\title{
VALORACIÓN DE RIESGOS INDUCIDOS POR LA CONSTRUCCIÓN DE NUEVOS EDIFICIOS EN EL CAMPUS DE LA UNIVERSIDAD DE DEUSTO. ESPAÑA
}

\section{(UNIVERSITY OF DEUSTO CAMPUS ENLARGEMENT: INDUCED HAZARDS EVALUATION. SPAIN)}

\author{
Jesús Saiz de Omeñaca, \\ Depto. de Ciencias e Ingeniería de la Tierra, el Terreno y los Materiales de la Universidad de Cantabria, \\ Avda. de los Castros, s/n. 39005 SANTANDER.
}

Iñaki Edeño, Ingenieria del Suelo Edeño, S. A., Elcano, 11, 48011 BILBAO.

José Antonio Saiz de Omeñaca, Depto. de Silvopascicultura, E.T.S.I. de Montes, Universidad Politécnica, 28040 MADRID

ESPAÑA

\section{RESUMEN}

El estudio de la morfologia de una cuenca adyacente a los terrenos donde se proyectaba construir un gran edificio, revelo que éste $y$ otras construcciones podrian resultar seriamente dañadas a causa de avenidas violentas. Para valorar tal riesgo, se calculó la cantidad de agua que en caso de precipitaciones extraordinarias caería en la cuenca, la permeabilidad de la misma, las posibilidades de retención por la vegetación, los suelos y los recubrimientos geológicos y diversos factores capaces de agravar la situación.

Se estudiaron posibles soluciones, eligiéndose la construcción de una galería de drenaje cuyo trazado y dimensiones garantizaba no solamente una correcta evacuación, sino también la posibilidad de que fuera sometida periódicamente a limpieza y unas mejores condiciones geotécnicas en la parte de la vaguada contigua a la construcción. Se realizaron también diversas recomendaciones, resaltándose en particular la importancia de conservar suelos y depósitos geológicos, así como el buen estado de la vegetación.

Realizada la obra y transcurrido un tiempo prudencial, la solución propuesta ha funcionado a la perfección, sin que se hayan presentado problemas no previstos.

\section{SUMMARY}

A study of the morphology of the basin adjacent to a projected construction site revealed that this building and others nearby could suffer serious damage in the event of flash flooding. In order to evaluate this risk, a number of factors were calculated; the amount of water that could fall onto the basin in case of extraordinary precipitation, the permeability of the solls and regoliths, the retention capacity of the vegetation, etc.

After studying a number of possible solutions, it was decided that a drainage gallery should be built. The dimensions and layout of this gallery would guarantee adequate drainage, make periodic cleaning possible and improve the geotechnic conditions in the area adjacent to the building site. A number of other recommendations were made with special emphasis on the importance of conserving the soils, regoliths and vegetation of the basin.

Now that the project has been completed and a period of time has passed, it can be asserted that the proposed solution has functioned perfectly without any unforeseen problems.

Palabras clave: Riesgos naturales, gestión del territorio, información de proyectos.

\section{INTRODUCCIÓN}

En 1985 recibimos el encargo de estudiar las posibles consecuencias derivadas de la ampliación de las edificaciones de la Universidad de Deusto, en la margen derecha de la ría de Bilbao. El informe realizado, que a continuación se resume, mostró que llevar a cabo las construcciones sin establecer un sistema de drenaje suficiente implicaba aceptar importantes riesgos, que podrían traducirse no solamente en daños más o me- 


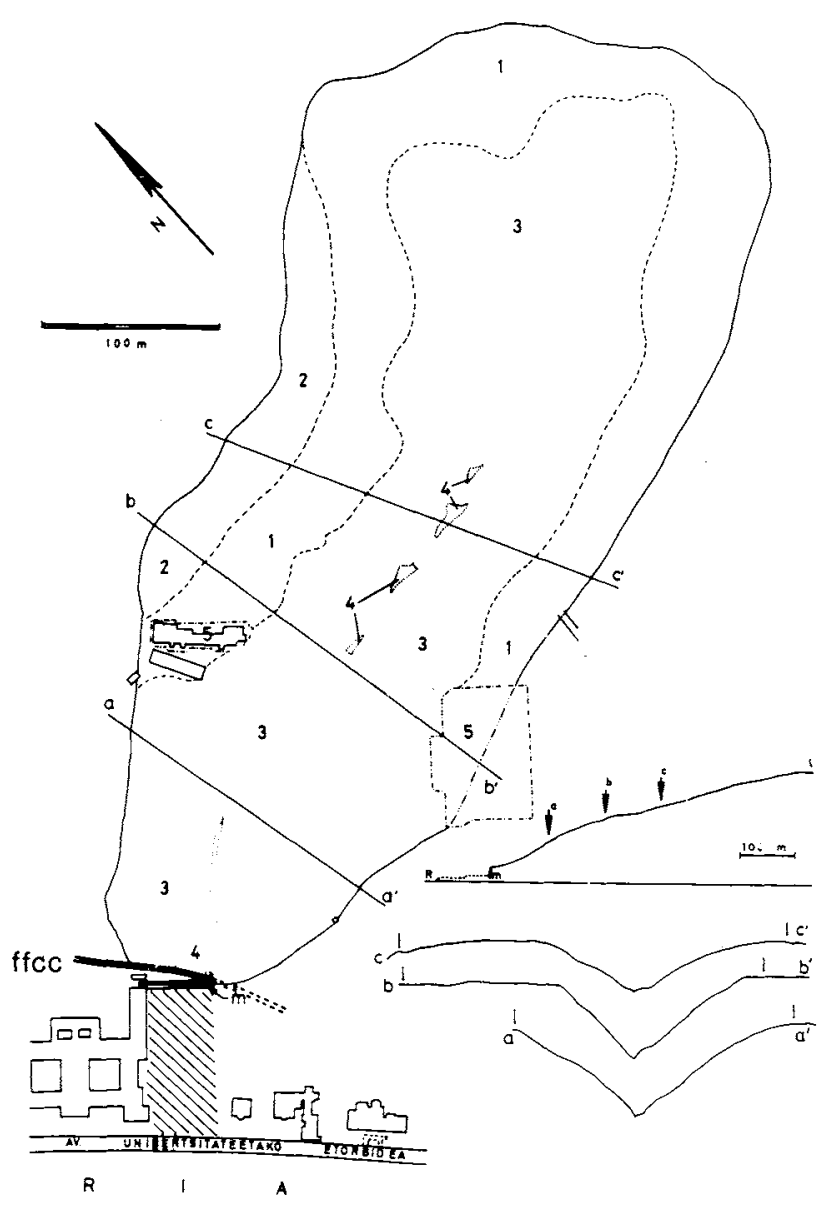

Fig. I.-Forma de la cuenca adyacente al campus de la Universidad de Deusto (delimitada por línea continua) y situación de la ria y de las vias del ferrocarril Las Arenas-Plencia (ffcc), del muro de contención ( $m$ ), de la zona de obra (rayado diagonal) y de la avenida mencio. nadas en el texto.

Los números 1 al 5 señalan la distribucion superficial de los principales recubrimientos. 1, Eluviones, 2 , id. dudoso. 3, depositos de ladera, 4 , rellenos de fondo de valle (depósitos aluviales). 5 , recubrimientos artificiales.

$a-a$, $b-b^{\prime}$, y $c-c^{\prime}$ son los perfiles transversales (sin exagerar el relieve) de la vaguada. $R \cdot m-t$, el perfil longitudinal (a escala más reducida), en el que se senala la situación de los perfiles transversales.

nos graves en los nuevos edificios, sino también en los antiguos, en el ferrocarril Las Arenas-Plencia en la Avenida del Dr. Morcillo y aun sobre otras actividades en la misma ría de Bilbao, con probable pérdida de vidas humanas.

Efectivamente, adyacente al campus se sitúa una vaguada que tiempo atrás desembocaba en la ría de Bilbao, precisamente a través de la zona de obra (Fig. I). En su parte terminal se realizaron, hace ya muchos años, diversas construcciones (vías de ferrocarril, muros y edificios) que implicaron una desaparición práctica de la capacidad de drenaje a través de la misma. Para obviar este inconveniente se contaba con sistemas artificiales de drenaje, incluida una galería subterránea, y con un cierto drenaje subsuperficial natural, que si bien eran suficientes en condiciones ordinarias suponían un riesgo importante en el caso de precipi- taciones intensas en un corto lapso de tiempo. Una clara manifestación de este riesgo se tuvo en agosto de 1983 , si bien en la zona concreta no hubo desgracias personales y los daños económicos fueron relativamen. te reducidos.

\section{MORFOLOGÍA DE LA CUENCA}

La cuenca hidrográfica de la vaguada muestra un perfil longitudinal con fuerte pendiente hacia la ría, pues pese a que la longitud es escasa $(600 \mathrm{~m})$ la cabecera se sitúa a más de $200 \mathrm{~m}$ por encima del nivel de las aguas en la misma (Cuadro I). Aproximadamente los dos tercios inferiores de la cuenca están recorridas por una pequeña (en condiciones ordinarias) corriente de carácter permanente, que nace de la confluencia de otras menores y que en la mayor parte de su trayecto corre semiencajada en un valle de trazado casi rectilineo, hasta terminar en el fondo de valle adyacente a las vías del ferrocarrill. Este valle corresponde geomorfológicamente al canal de desagüe de la cuenca y sus perfiles transversales muestran formas en $V$ muy marcadas, con unas relaciones profundidad/anchura muy altas (Cuadro II y fig. I). A ambos lados del valle en $\mathrm{V}$ y en la zona de cabecera se extiende una superficie aplanada, inclinada hacia la ría, que en parte vierte sus aguas de escorrentía hacia el valle.

\section{CUADRO I}

Altura sobre la Ría de Bilbao de los puntos que se indican, correspondientes a la vaguada estudiada

Parte superior de la cuenca .................... $>200 \mathrm{~m}$

Fondo de valle $400 \mathrm{~m}$ aguas arriba del ferro-

carril ................................................... $\quad 140 \mathrm{~m}$

Id. $300 \mathrm{~m}$................................................ $\quad 115 \mathrm{~m}$

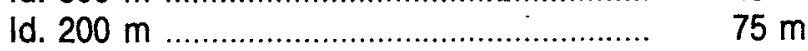

ld. $100 \mathrm{~m}$........................................................ $45 \mathrm{~m}$

Vía del ferrocarril ..................................... $27 \mathrm{~m}$

(Ver Foto 2). 
Al existir incertidumbre con respecto a si las precipitaciones sobre pequeñas zonas urbanizadas se vertian hacia la vaguada y a causa de deficiencias de la cartografía no fue posible fijar con exactitud el área de la cuenca, si bien en todo caso la extensión al norte de las vías del ferrocarril es como mínimo de $11,2 \mathrm{Ha}$, sobrepasando muy probablemente las 12,3.

El análisis de la forma, red de drenaje y topografía de detalle de la cuenca muestra sin lugar a dudas que en el caso de precipitaciones intensas las aguas de escorrentía de la cabecera de la misma tienden a concentrarse rápidamente en el fondo del valle, a unos $300 \mathrm{~m}$ aguas arriba de las vías del ferrocarril. Desde allí, y a través del canal de desagüe mencionado, se trasladan con gran rapidez hacia la ría, con lo que pueden originar los daños mencionados.

\section{MATERIALES QUE FORMAN LA CUENCA}

Con el fin de llegar a evaluar en la medida de lo posible el volumen de las aguas que podia llegar a concentrarse fue preciso establecer hasta qué punto podian infiltrarse y quedar retenidas en suelos, recubrimientos o depósitos superficiales (de donde se liberarian lentamente, pasarían a la atmósfera por evapotranspiración o ganarian zonas más profundas) o podian ser evacuadas rápidamente por posibles vías subterráneas naturales.
El examen de los materiales que forman la cuenca (Cua. dro III) mostró que las rocas del substrato, dispuestas en capas alternantes de composición y espesores variables, plegadas de manera más o menos apretada, presentan en conjunto una muy baja permeabilidad, por lo que las salidas de agua de la cuenca por vía subterránea son muy bajas. Es decir, el substrato rocoso (para más detalles sobre el mismo cfr. 1) forma un auténtico vaso hidrológico, a través del cual el agua escapa muy lentamente. Por ello, en el caso de precipitaciones extraordinarias, la mayor parte de las aguas tendrian que salir de la vaguada de alguna otra forma 0 tendrian que retenerse eficazmente para que la infiltración, la evaporación, la transpiración y la escorrentía pudieran evacuarlas.

En las partes no urbanizadas, las rocas de la vaguada están recubiertas en más del $98 \%$ por materiales detriticos incoherentes formados por arcillas, limos, arenas y fragmentos de rocas diversas originados por meteorización de las mismas rocas de la zona. En el Cuadro III se señalan los distintos tipos de recubrimien. tos y depósitos existentes en la vaguada, así como los resultados de calcular la superficie y el volumen que ocupan.

Es importante señalar que los depósitos de ladera están sometidos a procesos geológicos activos que implican desplazamientos gravitatorios a favor de la pendiente. Por otra parte, la composición, incoherencia y situación en las laderas (ocasionalmente con ángulos

\section{CUADRO III \\ Resumen sobre los materiales de la cuenca hidrográfica}

Rocas del substrato

Margas

Calizas

Areniscas

Limolitas y otras

Recubrimientos y depósitos geológicamente recientes

Eluviones (2)

Superficie ocupada (1)

$46.300 \mathrm{~m}^{2}$

$67.700 \mathrm{~m}^{2}$

$1.000 \mathrm{~m}^{2}$

(3)

(4)

$700 \mathrm{~m}^{2}$

Suelos

$2.700 \mathrm{~m}^{2}$
Volumen (1) $30-40.000 \mathrm{~m}^{3}$ $20-30.000 \mathrm{~m}^{3}$ $1.200-1.400 \mathrm{~m}^{3}$

NOTAS:

1. Medidas correspondientes a la parte de la cuenca aguas arriba del FFCC.

2. Incluye zonas dudosas.

3. No calculado por estar al sur de las vías del ferrocarril

4. Incluidos con los materiales que recubren. 
de reposo cercanos a los límites de estabilidad) hace a estos depósitos muy vulnerables, por lo que por erosión pueden ser arrastrados parcialmente por las aguas de escorrentía superficial. En el caso de precipitaciones intensas, esto puede dar lugar a que las aguas arrastren una carga de sedimentos importante, originándose un líquido de densidad superior a la del agua, capaz de agravar los problemas que ya se han mencionado. Además, el comportamiento mecánico de tales depósitos es también muy deficiente y en particular

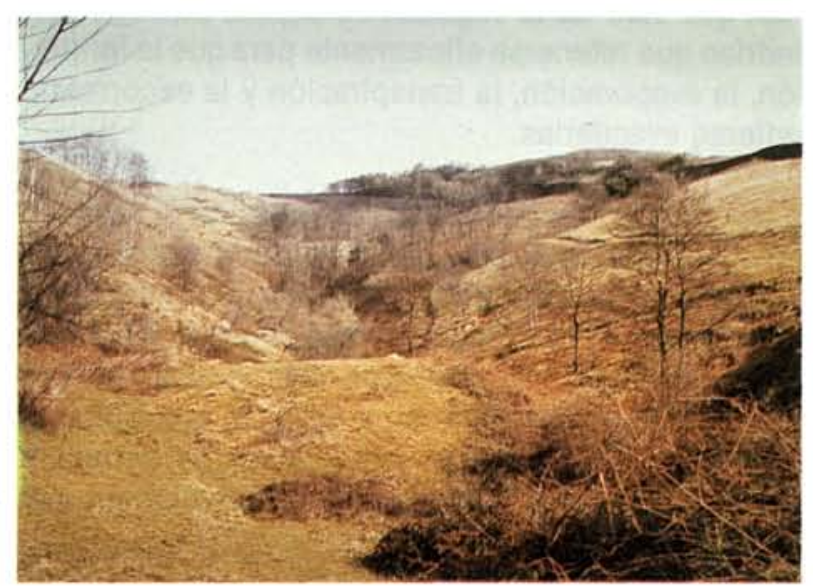

1

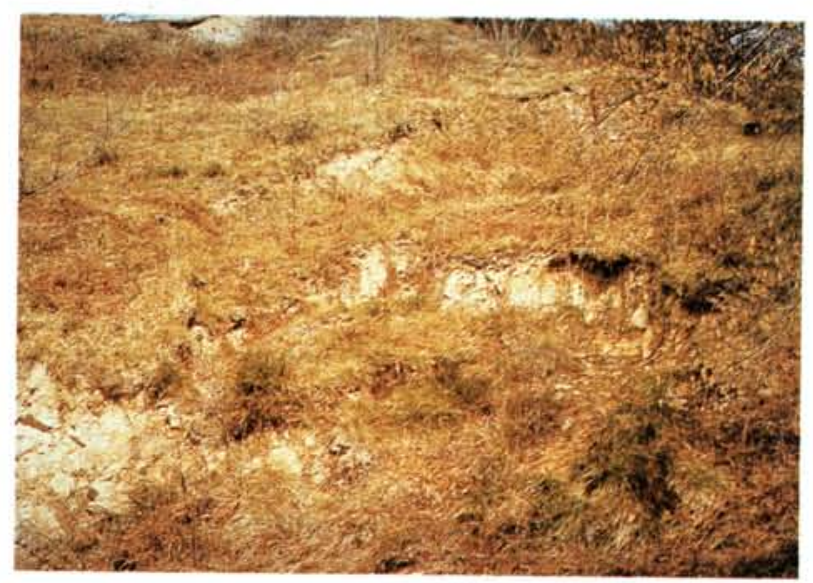

2

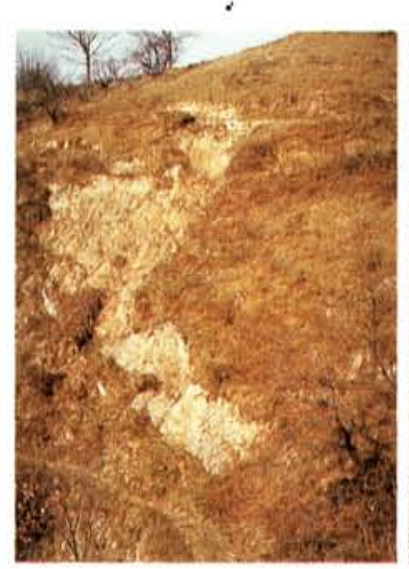

3

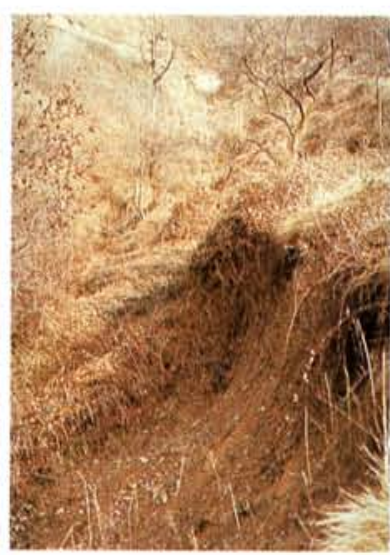

4 puede dar lugar a una capacidad portante extremadamente baja.

También los depósitos de fondo de valle (cuyo espesor es casi siempre bastante reducido, pues sólo inmediatamente al norte de las vías del ferrocarril alcanzan más de $8 \mathrm{~m}$ en su parte media, sin superar, probablemente, los 9) están superficialmente amenazados por riesgos de erosión aterramiento, siendo su capacidad portante moderada.

1) Zona de cabecera de la cuenca. En las partes allanadas o con escasa pendiente se han formado eluviones aunque por excepción, en la que aparece en primer término, los materiales deben corresponder a un antiguo fondo de valle. Donde la pendiente es mayor, los recubri. mientos son del tipo depósitos de ladera, aunque con un marcado carácter eluvionar. En el centro de la fotografía puede apreciarse la existencia de un escarpe producido por un deslizamiento de ladera de reducidas dimensiones.

Como puede verse, la vegetación cubre la práctica totalidad de la ca. becera, predominando la herbácea y, en menor medida y en algunas partes, la arbustiva o la arbórea.

2) Esta fotografia y las dos siguientes ilustran los procesos geológi cos activos a escala humana que afectan directamente a los depósitos de ladera definidos en el texto, con imágenes de la zona por la que la tubería del consorcio atraviesa al canal de desagüe de la cuenca. Los procesos más llamativos son dos deslizamientos "en golpe de cuchara", que se han traducido claramente en terreno disturbado y en sendos escarpes. Además, las zonas no cubiertas por vegetación están sometidas a procesos de erosión acelerada y existen también señales diversas que parecen demostrar la existencia local de reptación.

3) La inestabilidad de los depósitos de ladera se manifiesta aqui claramente en los fenómenos de erosión acelerada, que pueden llegar a hacer peligrar seriamente a la tuberia.
4) Descarne reciente sometido a erosión acelerada. Puede observarse cómo la vegetación, pese a la pendiente, pobreza del "suelo", etc., ha comenzado a colonizarlo, de tal forma que es de esperar que en poco tiempo los materiales incoherentes queden protegidos. Al fondo, alcantarilla bajo la tubería anteriormente mencionada. Esta foto. grafia, con la tercera y la séptima, y otras en menor medida, ilustra hasta qué punto la conservación de la vegetación es importante, tal y como se expresa en el texto. 
Más del $95 \%$ de la superficie de la vaguada no urbanizada está recubierta por suelos, asentados generalmente sobre eluviones, fondos de valle y depósitos de ladera. En los dos primeros casos suelen tener un desarrollo aceptable, pero en el tercero se trata con frecuencia de suelos poco evolucionados, con un horizonte $\mathrm{A}$ reducido, muy lavados, con escasa materia orgánica y texturalmente deficientes, cercanos a litosoles. Estos suelos, como los depósitos de ladera sobre los que se asientan, son muy vulnerables y además tienen una ca-

5) No solamente los deslizamientos en "golpe de cuchara" y la ero. sión amenazan a la tubería del consorcio. En el lugar en que ésta atraviesa el cauce se ejerce un efecto de represado y la sección y cons. trucción de la alcantarilla para el paso de la corriente parecen inade. cuados.

6) Los pequeños obstáculos en el cauce contribuyen a que la esco. rrentía superficial sea más lenta y retienen sedimentos, con lo que aparecen rellenos del tipo fondo de valle. La rapidez con que se forman ilustra la carga sólida que llevan las corrientes y la velocidad con que se llenarian unas hipotéticas presas de regulación. pacidad de retención de agua más reducida que los suelos bien desarrollados.

Los recubrimientos y depósitos geológicamente recientes se estudiaron con detalle debido a que podian retener cantidades importantes de agua, que de otra forma se sumarian a la escorrentía superficial; y a que podian agravar y aun originar problemas geotécnicos diversos (cfr., por ejemplo, (6), II.6; (3), c. XIV).
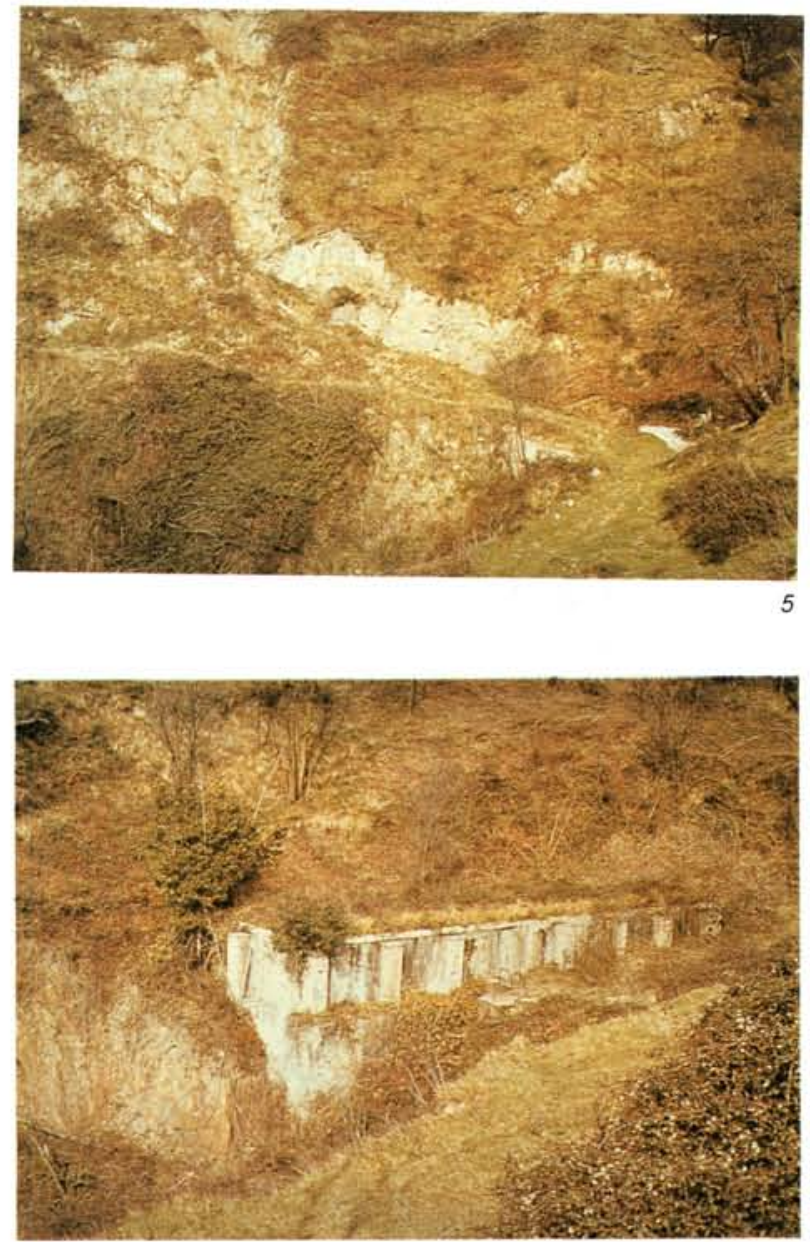

6

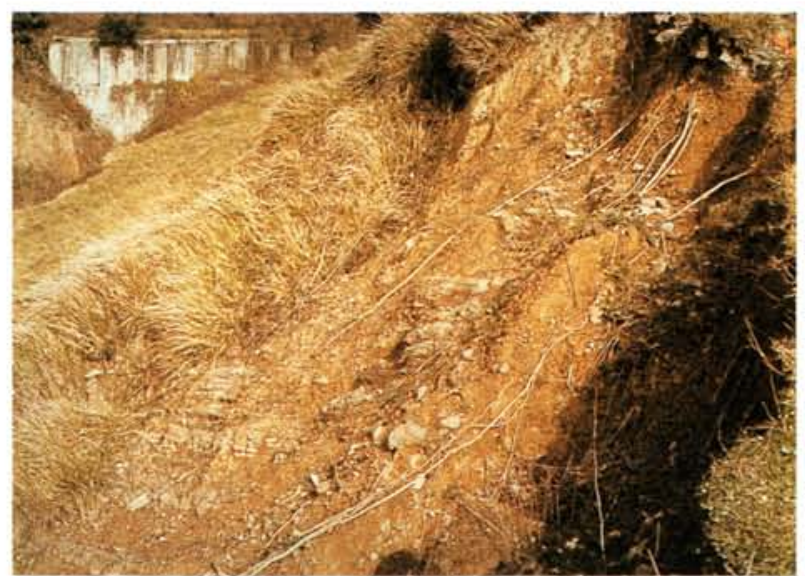




\section{PAPEL DE LA VEGETACIÓN}

La cuenca en estudio aparecía cubierta en más de un $95 \%$ por vegetación, predominando los prados en las partes altas allanadas y en las de suaves pendientes y las especies herbáceas y arbustivas, en ocasiones tipo landa, en las laderas empinadas. En ciertas partes, sobre todo en lugares protegidos, se ha implantado una vegetación en la cual el estrato dominante llega a ser claramente el arbóreo.

La importancia de la vegetación de cara a los problemas que se planteaban en la zona es muy importante y se debe fundamentalmente, a escala humana, a las acciones siguientes (para más detalles, cfr., por ejemplo (6), II.3):

- Intercepta parte de las precipitaciones, que al quedar mojando hojas, tallos, ramas, etc., o bien no llegan al suelo o lo hacen más espaciadamente.

- Obstaculiza, mediante la acción de sus partes aéreas o de sus raíces, la remoción de partículas debida al impacto de las gotas de lluvia, a la acción del viento, a las aguas no encauzadas, a las corrientes efímeras, etcétera.

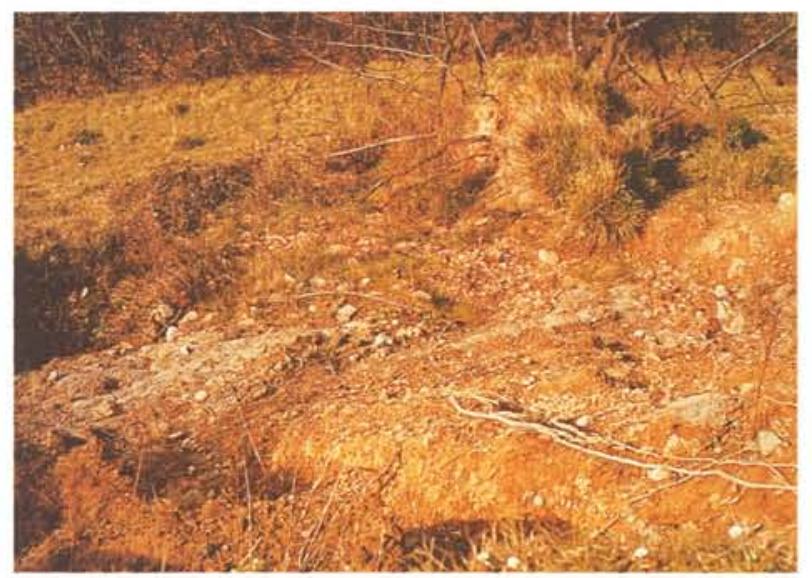

8

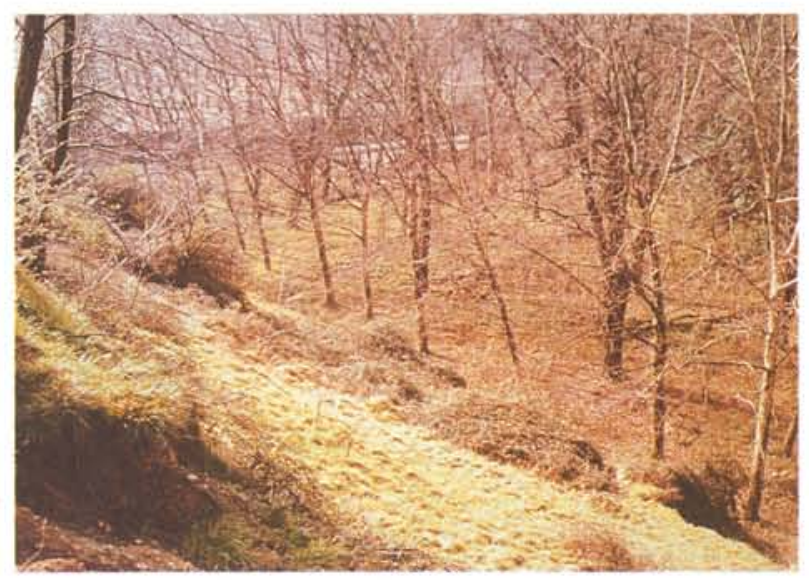

9
- Frena la escorrentía superficial, haciéndola más lenta y aumenta el poder de infiltración del suelo, lo que contribuye a disminuir el flujo superficial. Estas acciones tienen enorme importancia en el caso de precipitaciones extraordinarias.

- Absorbe parte del agua del suelo y del subsuelo, transpirándola a la atmósfera.

- Da lugar a la existencia y permite la dinámica de los suelos, que retienen agua, protegen de la erosión a recubrimientos y depósitos incoherentes y, a su vez, permiten el desarrollo y buen estado de la vegetación.

Este conjunto de acciones se traduce tanto en una caida en el volumen de la escorrentia superficial total como en una fuerte disminución del riesgo de avenidas violentas. Algunas de aquellas acciones pueden tener en este caso un efecto directo pequeño (por ejemplo, se necesita tiempo para que el volumen de agua transpirada sea importante y evidentemente es pequeño en el caso de días lluviosos), pero contribuyen eficazmente a evitar que suelos, aluviones, depósitos de ladera y fondos de valle estén saturados de agua, por lo que pueden absorber y retener un volumen importante con

8) Un cerezo desarraigado y caído, cuyo cepellón ha sido cubierto por gramineas, originó una zona sin protección que, posteriormente, se fue ampliando hasta amenazar al camino. Nótese que, en algunas par. tes, la roca del subsuelo ha llegado a quedar al descubierto. Si "cica. trices" como ésta no fueran colonizadas rápidamente por la vegeta. ción, su ampliación progresiva originaria serios problemas.
9) El relleno del fondo de valle adyacente a las vias del ferrocarril es el más voluminoso de la cuenca. Debe su existencia, precisamente, al muro de contención, que ha provocado una caida en la velocidad de las aguas superficiales en ese lugar y, por tanto, un aumento de la carga sobre el mismo muro, mayores condiciones de humedad, etcétera. 
ocasión de precipitaciones intensas. Todo parece indicar que la vegetación de la vaguada ha jugado, juega y debiera seguir jugando un importantísimo papel, evitando muy probablemente que en agosto de 1983, y quizá en otras ocasiones, ocurrieran verdaderas catástrofes.

Otra consecuencia importante que se deriva de la existencia y buen estado de la vegetación es que se protege a suelos y depósitos y recubrimientos incoherentes frente a la erosión. Esto es importante no sólo porque contribuyen a retener volúmenes importantes de agua, sino también porque se evita que el material erosionado y arrastrado por las aguas origine $\mathrm{o}$ agrave problemas diversos (avenidas de agua y lodos, aterramiento de desagües artificiales, mayor carga sobre el muro de contención al sur de las vías del ferrocarril, etc.). Afortunadamente, si bien es cierto que un suelo erosionado o degradado tarda mucho tiempo en rehabilitarse, bajo el clima y las condiciones locales crece fácilmente una cubierta vegetal mínima incluso en materiales que no parecen merecer el calificativo de suelos.

En resumen, la conservación en buen estado de la vegetación en la vaguada es de primera importancia si

10) Efectos debidos a las aguas de escorrentia superficial en agosto de 1983, al norte de las vias del ferrocarril. se quieren minimizar diversos riesgos naturales, particularmente el de avenidas violentas. Si alguna vez esta vegetación ha de desaparecer, en todo o en parte, será preciso prever unos desagües comparativamente mayores y más seguros, capaces de evacuar el agua en lapsos de tiempo inferiores, asi como vigilar su posible aterramiento y, en su caso, realizar periódicas labores de limpieza.
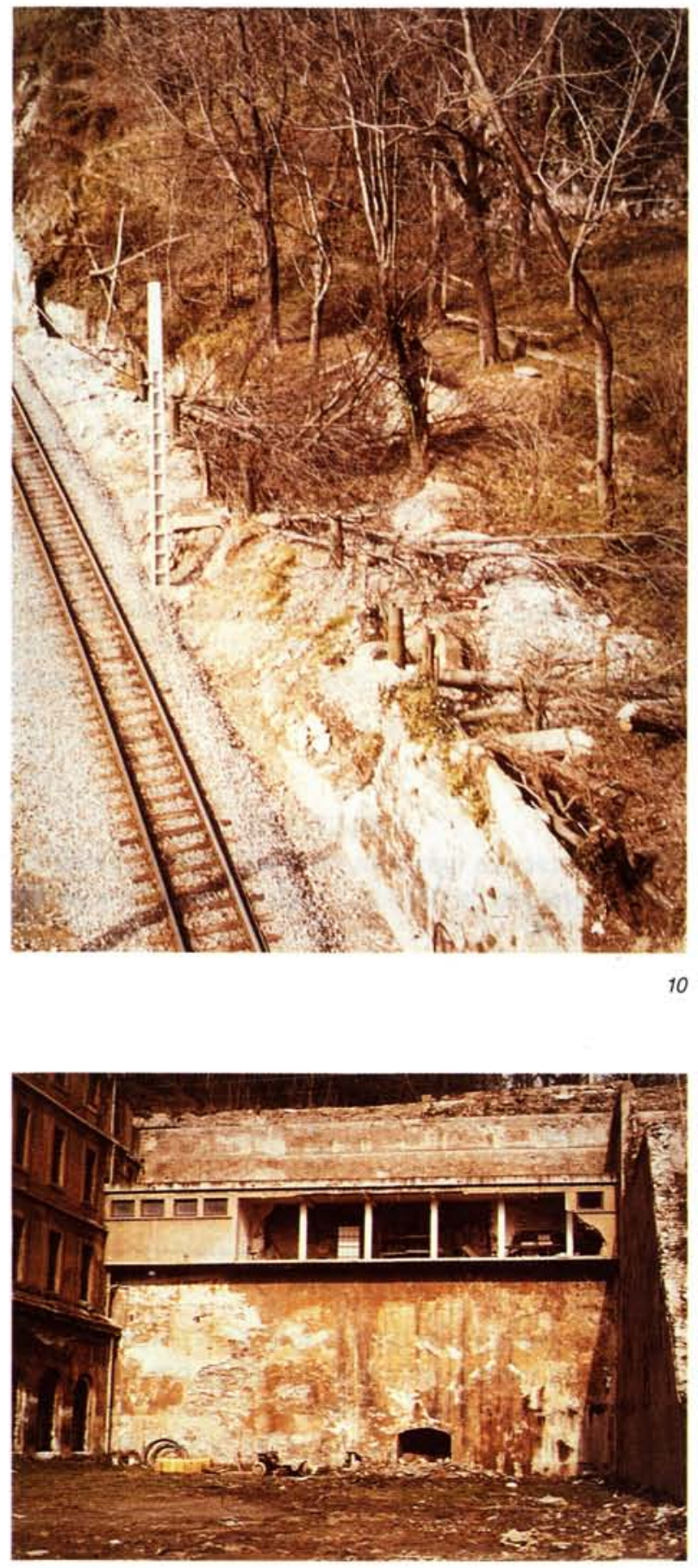

http://informesdelaconstruccion.revistas.csic.es
11) Muro de contención, desde su parte sur. Los rellenos artificiales y naturales han elevado considerablemente el perfil transversal del antiguo valle. La utilización de la galeria cuya desembocadura se pue. de observar hacia abajo y a la derecha del centro de la fotografia, ten. dria, entre otras ventajas, la de su situación respecto al perfil de aquel antiguo valle, ya que permitiria un mejor drenaje de los rellenos adya. centes.

(C) Consejo Superior de Investigaciones Científicas

Licencia Creative Commons 3.0 España (by-nc) 


\section{ESCORRENTIA SUPERFICIAL Y RIESGO DE AVENIDAS}

En el caso que nos ocupa, las precipitaciones ordinarias no originan problemas, pero sí las lluvias son extraordinarias (y este es el caso que debe considerarse, por ser el que supone mayores riesgos); la situación cambia drásticamente al alcanzarse el limite máximo de capacidad de almacenamiento de agua en los recubrimientos. En estas circunstancias, la casi totalidad de las precipitaciones se transforma en aguas de escorrentía superficial y sólo cuando las lluvias desaparecen o se hacen mínimas pueden los suelos comenzar a perder parte del agua infiltrada.

Según los datos que nos fueron facilitados, que concuerdan con las estimaciones de (7) (Cuadro IV), en la zona no son excesivamente raras las precipitaciones del orden de 50 a $100 \mathrm{l} / \mathrm{m}^{2} /$ dia y se han llegado a registrar 160. Esto significa que cabe esperar que caigan sobre la cuenca con cierta frecuencia entre 6.000 y $12.500 \mathrm{~m}^{3}$ en 24 horas y excepcionalmente habria que contar con cifras del orden de $20.000 \mathrm{~m}^{3}$ o incluso superiores si se quiere eliminar totalmente el riesgo de avenidas violentas. Además, la distribución de las precipitaciones no es uniforme a lo largo de la jornada y podría concentrarse en lapsos de tiempo menores o darse precipitaciones extraordinarias durante un tiempo superior. La importancia de la vegetación, que tiende a repartir las aguas durante espacios de tiempo más prolongados, queda así de manifiesto. También alguno de los obstáculos existentes actualmente en el cauce dan lugar a un cierto retardo, pero su efectividad real es muy reducida.

\section{CUADRO IV}

Precipitaciones máximas en el entorno del campus de la Universidad de Deusto. Cuadro elaborado a partir de

(7)

\begin{tabular}{|c|c|c|}
\hline $\begin{array}{c}\text { Tiempo de precipitación } \\
\text { (horas) }\end{array}$ & $\begin{array}{c}\text { Periodo de retorno } \\
\text { (años) }\end{array}$ & $\begin{array}{c}\text { Precipilación máxima } \\
\text { (mm) }\end{array}$ \\
\hline 1 & 10 & 50 \\
6 & 10 & 70 \\
12 & 10 & 100 \\
24 & 10 & 100 \\
24 & 50 & 140 \\
24 & 100 & 140 \\
\hline
\end{tabular}

Dada la premura de tiempo (prácticamente se habian finalizado los movimientos de tierras previos a la obra propiamente dicha y retrasar ésta hubiera significado importantes costes) y la indole del problema, no se justificaban complejas y detalladas mediciones de campo para determinar la capacidad de acumulación por infiltración, pues ésta puede también estimarse a partir de las características de composición y textura y del volumen de suelos y recubrimientos (6, pp. 166-183), aunque los márgenes de seguridad deban ser amplios. Así, ya que la cantidad máxima de agua que puede almacenarse en tales materiales depende de su porosidad (dato que puede estimarse a partir de tablas existentes en numerosos manuales, como (4); (5); (2), etc.), según nuestros cálculos, la capacidad límite de almacenamiento podía alcanzar como máximo del orden de los 8.000 a los $14.000 \mathrm{~m}^{3}$. No obstante, estas cifras pueden resultar engañosas, pues en las condiciones de esta cuenca el volumen de agua que realmente puede retenerse es mucho menor y depende de factores en gran medida aleatorios e impredecibles para un momento determinado, como el grado previo de saturación. Es más, es difícil, debido a causas diversas (naturaleza de la matriz de suelos y recubrimientos, factores climatológicos,...) que ese grado sea muy bajo y por ello estimamos que la capacidad real máxima de retención puede alcanzar entre 5.000 y $8.000 \mathrm{~m}^{3}$ tras un periodo sin lluvias relativamente largo y puede ser prácticamente nula después de varios días con lluvias importantes. En términos generales, es de esperar que la capacidad real de retención sea relativamente alta desde agosto a noviembre o diciembre y pequeña desde febrero a mayo, pero puede haber desviaciones importantes.

Teniendo en cuenta los datos anteriores, puede afirmarse que en el caso más desfavorable habría que contar con precipitaciones capaces de producir un caudal de hasta $20.000 \mathrm{~m}^{3} / \mathrm{dia}$ <ó 25.000 si se quieren extremar las precauciones), con una capacidad efectiva de retención por los recubrimientos nula y solamente con la acción, importante, de retardo debido a la vegetación.

\section{DISCUSIÓN Y CONCLUSIONES}

Resultaba indiscutible la necesidad de un sistema o sistemas capaces de evacuar hasta unos 20.000 ó $25.000 \mathrm{~m}^{3}$ en 24 horas (231,5 ó 289,3 litros por segundo), por lo que el desagüe existente era inadecuado, siendo además su trazado no idóneo y careciendo probablemente de sección suficiente en toda su longitud (no pudo comprobarse este extremo).

La mejor solución a este problema consistía en utilizar una perforación existente en la parte baja del muro de contención entre las vías del ferrocarril y el campus de la Universidad. Su sección era más que adecuada, podía acondicionarse para que actuara como desagüe, su posición era geológicamente casi idónea (cercana a la parte inferior de los rellenos del antiguo valle en $\checkmark$ que existía en la zona antes de la construcción del muro de contención mencionado) y podia continuarse bajo los futuros edificios hasta desembocar por debajo de la avenida del Dr. Morcillo en la ría del Nervión con un ángulo apropiado. Una ventaja adicional, y no pequeña, era que resultaba posible mejorar las condi- 
ciones del muro de contención, al menos si la obra se efectuaba de tal forma que drenase también las aguas de la parte posterior del mismo (por ejemplo, realizando un revestimiento de seguridad discontinuo). Efectivamente, el mejor drenaje de las rocas y del fondo de valle inmediatos daría lugar a una disminución de la carga sobre el muro, a menores filtraciones, etc. El principal inconveniente era que el reacondicionamiento debería llevarse a cabo con las debidas precauciones, dado que las caracteristicas de la roca y de los depósitos hacian prever la aparición de problemas geotécnicos.

Otras posibles soluciones se revelaron como menos adecuadas o monetariamente prohibitivas. Por ejemplo, aumentar o substituir la vegetación por otra más adecuada sólo proporcionaría una solución parcial, incrementaría los riesgos durante el proceso y sus efectos reales serian difíciles de cuantificar a priori; construir un pequeño embalse (o más de uno), que probablemente se rellenaria con rapidez, seria excesivamente costoso; establecer otro tipo de obstáculos en el cauce no sería por sí solo suficiente y debería realizarse de tal forma que su superación o ruptura no significara un riesgo; cambiar la topografía de detalle sería una labor excesivamente costosa (no sólo en términos monetarios), con ciertos riesgos y de resultados relativamente pobres, dada la baja permeabilidad de las rocas del substrato, etcétera.

El informe final destacaba la enorme importancia del buen estado de la vegetación en la vaguada y como el desarrollo urbanístico en cualquier parte de ésta puede implicar variaciones importantes en la escorrentia superficial (8) si no se establecía un drenaje y alcantarillado adecuados.

Otros problemas menores también merecieron un comentario específico. Por ejemplo, con respecto a la cimentación de los edíficios proyectados en la zona baja, aparte de salvar las dificultades inherentes a los materiales que recubren a las rocas del subsuelo en el lugar (elevada plasticidad, baja capacidad portante, expansividad, etc., que según la información recibida ya se había previsto y se había actuado en consecuencia preparando una cimentación adecuada), resultaba aconsejable no obstaculizar e incluso favorecer el drenaje subsuperficial. Efectivamente, el comportamiento geotécnico de aquellos materiales es más favorable si su contenido en agua, que puede llegar a ser muy alto, es reducido y no sufre variaciones importantes. Por lo mismo resultaba también aconsejable establecer un sistema que mejorara la evacuación de agua subsuperficiales de la zona.

La estructura en delgadas capas en las que alternan materiales diferentes, muy plegadas, asi como la com. posición, rica en materiales arcillosos y limolíticos, también podía originar riesgos. El más grave, un posible deslizamiento, a favor de una estructura desfavo. rable, de parte de esos materiales junto a la nueva construcción, donde se preveía una pendiente elevada. Resultaba adecuado aconsejar que se evitase ese riesgo, bien mediante algún sistema de anclaje bien investigando más a fondo su posible incidencia.

Finalmente, también se denunciaba la situación de una conducción de suministro de aguas, que atravesaba el canal de desagüe natural de la cuenca en su parte superior. Localmente, estaba siendo puesta al descubierto por la erosión; cruzaba una zona particularmente sometida a deslizamientos y reptación superficial; la sección y construcción de la alcantarilla inferior en el cauce parecia inadecuada, etcétera.

\section{EPILOGO}

Una vez realizada la obra y transcurrido un tiempo prudencial, ha podido constatarse que la adopción de la solución propuesta para resolver el principal riesgo planteado ha funcionado a la perfección, sin que se haya presentado problema adicional alguno.

\section{AGRADECIMIENTOS}

Los autores agradecen al Dr. D. Angel Ramos su consejo y la crítica del manuscrito.

\section{BIBLIOGRAFIA}

(1) ADARO. Mapa geológico de España 1:50.000. BILBAO (61). Instituto Geológico y Minero de España, Madrid, 1975.

(2) Bowen, R. Groundwater. Elsevier, Amsterdam, 1986.

(3) Campy, M. et Macaire, J. J. Géologie des formations superficielles. Masson, París, 1989.

(4) Castany, G. Traité practique des eaux subterraines. Dunod, Paris, 1967.

(5) Custodio, E. y Llamas, M. R. Hidrología subterránea, Tomo I. Omega, Barcelona, 1976.

(6) Dunne, Th. \& Leopold, L. B. Water in environmental planning. Freeman, S. Francisco, 1978.

(7) Elias Castillo, F. y Ruiz Beltrán, L. Precipitaciones máximas en España. Estimaciones basadas en métodos estadisticos. Ministerio de Agricultura, Monografías ICONA 21, Madrid, 1979.

(8) Leveson, D. Geology and the urban environment. Oxford Univ. Press, New York, 1980. 